\title{
Identification of an Oleanane-Type Triterpene Hedragonic Acid as a Novel Farnesoid X Receptor Ligand with Liver Protective Effects and Anti-inflammatory Activity $\$$
}

\author{
Yi Lu, Weili Zheng, Shengchen Lin, Fusheng Guo, Yanlin Zhu, Yijuan Wei, Xi Liu, \\ Shikai Jin, Lihua Jin, and Yong Li \\ State Key Laboratory of Cellular Stress Biology, School of Life Sciences, Innovation Center for Cell Signaling Network, Xiamen \\ University, Xiamen, Fujian, China
}

Received July 13, 2017; accepted November 15, 2017

\begin{abstract}
Farnesoid $X$ receptor (FXR) and G-protein-coupled bile acid receptor 1 (GPBAR1) are two important bile acid (BA) receptors. As non-BAs drug template for GPBAR1, none of the natural oleanane-type triterpenes have been reported as FXR ligands, despite FXR and GPBAR1 having similar binding pockets for BAs. Here, we report the natural triterpene hedragonic acid that has been isolated from the stem and root of Celastrus orbiculatus Thunb. (COT) as an effective agonist for FXR. Both biochemical amplified luminescent proximity homogeneous assay and cellbased reporter assays showed that hedragonic acid regulated the transcriptional activity of FXR. Circular dichroism spectroscopy further suggested the conformational changes of FXR upon the binding of hedragonic acid. Interestingly, the crystal structure
\end{abstract}

of hedragonic acid-bound FXR revealed a unique binding mode with hedragonic acid occupying a novel binding pocket different from the classic binding position. The structural comparison between hedragonic acid-bound FXR and oleanolic acid-bound GPBAR1 explained the molecular basis for the selectivity of oleanane-type triterpenes for FXR. Moreover, hedragonic acid treatment protected mice from liver injury induced by acetaminophen overdose and decreased hepatic inflammatory responses in an FXR-dependent manner, suggesting that hedragonic acid might be one of the major components of COT for its multifunctional pharmaceutical uses. In conclusion, our results provide novel structure templates for drug design based on natural triterpenes by targeting FXR and/or GPBAR1 with pharmaceutical values.

\section{Introduction}

Farnesoid X receptor (FXR) and G-protein-coupled BA receptor 1 (GPBAR1) are two important bile acid (BA) receptors. FXR, a member of the nuclear hormone receptor superfamily, is highly expressed in mammalian liver, intestine, kidney, and adrenal gland (Makishima et al., 1999; Parks et al., 1999). Small molecules known as ligands play important roles in modulating the activity of nuclear receptors (Benet et al., 2015; McKenna, 2016). Similar to many other nuclear receptors, ligand binding induces the conformational change of FXR, leading to the modulation of its transcriptional

The authors declare no competing financial interest.

This work was supported by grants from the National Natural Science Foundation of China [U1405220, 81471084, 81773793, 31770814, and 31470726]; the Fundamental Research Funds for the Central Universities [20720150052]; the Programme of Introducing Talents of Discipline to Universities [B12001]; and the National Science Foundation of China for Fostering Talents in Basic Research [J1310027].

https://doi.org/10.1124/mol.117.109900.

S This article has supplemental material available at molpharm. aspetjournals.org. function through the recruitment or release of specific coregulators, including coactivators such as the steroid receptor coactivator (SRC) family, and corepressors such as the nuclear corepressor (NCoR) (Jin and Li, 2010). As a BA receptor, FXR plays important roles in maintaining bile acid homeostasis (Kong et al., 2012; Matsubara et al., 2013; Sun et al., 2017). Moreover, FXR regulates many physiologic functions, including metabolism, cancer, and liver regeneration (Wagner et al., 2011). Notably, FXR-null mice display strong hepatic inflammation and develop spontaneous liver tumors (Yang et al., 2007; Wang et al., 2008). Interestingly, FXR ligands have shown anti-inflammatory activity and liver protective effects by targeting the FXR pathway (Lee et al., 2010; Meng et al., 2010; Bhushan et al., 2013). GPBAR1 is the membrane-bound BA receptor (Maruyama et al., 2006; Keitel and Häussinger, 2012) that is also highly expressed in the liver and intestine, as well as in brown adipose tissue and the spleen (Keitel and Häussinger, 2012). Similar to FXR, GPBAR1 plays important roles in regulating energy homeostasis and glucose metabolism (Thomas et al., 2009; Broeders et al., 2015). Activated

ABBREVIATIONS: AlphaScreen, Amplified Luminescent Proximity Homogeneous Assay; ALT, alanine aminotransferase; APAP, acetylpara-aminophenol; AST, aspartate aminotransferase; BA, bile acid; CD, circular dichroism; CDCA, chenodeoxycholic acid; COT, Celastrus orbiculatus Thunb.; FXR, farnesoid X receptor; GPBAR1, G-protein-coupled bile acid receptor 1; GW4064, 3-(2,6-Dichlorophenyl)-4-(3'-carboxy-2chlorostilben-4-yl)oxymethyl-5-isopropylisoxazole; LBD, ligand-binding domain; LDH, lactate dehydrogenase; NCoR, nuclear corepressor; OCA, obeticholic acid; PCR, polymerase chain reaction; PDB ID, Protein Data Bank identifier; SRC, steroid receptor coactivator; TC-100, $3 \alpha, 7 \alpha, 11 \beta$ trihydroxy-6 $\alpha$-ethyl-5 $\beta$-cholan-24-oic acid. 
GPBAR1 induces the production of glucagon-like peptide-1, which in turn modulates insulin secretion/sensitivity, glucagon secretion, and $\beta$-cell mass (Pellicciari et al., 2009). In addition, binding of BAs with GPBAR1 increases energy expenditure in brown adipose tissue, preventing obesity and insulin resistance (Watanabe et al., 2006; Broeders et al., 2015). Thus, both FXR and GPBAR1 have been promising drug targets for treating a variety of diseases affecting liver, intestine, and kidney.

Many natural bile acids, such as cholic acid, chenodeoxycholic acid (CDCA), lithocholic acid, and deoxycholic acid, are all ligands for both FXR and GPBAR1 (Porez et al., 2012). However, some BAs or semisynthetic BA derivatives selectively recognize only FXR or GPBAR1. For example, obeticholic acid (OCA; INT-747; 6-ECDCA) and TC-100 $(3 \alpha, 7 \alpha, 11 \beta$ trihydroxy- $6 \alpha$-ethyl-5 $\beta$-cholan-24-oic acid) are potent FXR agonists (Fiorucci et al., 2009; Pellicciari et al., 2016). INT-777 (S-EMCA) and 23(S)-methylated lithocholic acid selectively activate GPBAR1, whereas INT-767 is an agonist for both BA receptors (Fiorucci et al., 2009; Yu et al., 2015; Sun et al., 2017). Aside from BAs, a class of plant natural triterpenes, including betulinic acid, oleanolic acid, and ursolic acid, were identified as selective GPBAR1 agonists with physiologic functions (Sato et al., 2007; Genet et al., 2010). These natural oleanane-type triterpenes have been non-BA drug templates for target GPBAR1 (Genet et al., 2010; Castellano et al., 2013). Even though FXR and GPBAR1 have similar binding pockets for BAs, none of the oleanane-type triterpenes have been reported as FXR ligands. In this study, we identified hedragonic acid (24-nor-3-oxo-12-oleanen-28-oic acid), a natural pentacyclic oleanane-type triterpene that has been isolated from the stem and root of Celastrus orbiculatus Thunb. (COT), as an effective agonist of nuclear receptor FXR with pharmaceutical potentials.

\section{Materials and Methods}

Protein Preparation. The human FXR ligand-binding domain (LBD; residues 243-472) was expressed as N-terminal 6× His fusion protein from the expression vector pET24a (Novagen, Madison, WI). BL21 (DE3) cells transformed with expression plasmids were grown in Luria-Bertani broth at $25^{\circ} \mathrm{C}$ to an $\mathrm{OD}_{600}$ of $\sim 1.0$ and induced with $0.1 \mathrm{mM}$ isopropyl 1-thio- $\beta$-D-galactopyranoside at $16^{\circ} \mathrm{C}$. Cells were harvested and sonicated in $200 \mathrm{ml}$ of extraction buffer $[20 \mathrm{mM}$ Tris $(\mathrm{pH}$ $8.0), 150 \mathrm{mM} \mathrm{NaCl}, 10 \%$ glycerol, and $25 \mathrm{mM}$ imidazole] per $6 \mathrm{l}$ of cells. The lysate was centrifuged at $20,000 \mathrm{rpm}$ for 30 minutes, and the supernatant was loaded on a $5 \mathrm{ml} \mathrm{NiSO}$-loaded HiTrap HP column (GE Healthcare, Piscataway, NJ). The column was washed with extraction buffer, and the protein was eluted with a gradient of 25$500 \mathrm{mM}$ imidazole. The FXR LBD was further purified with a Q-Sepharose column (Amersham Biosciences, Uppsala, Sweden). To prepare the protein-ligand complex, we added a 5 -fold molar excess of hedragonic acid to the purified protein, followed by filter concentration to $10 \mathrm{mg} / \mathrm{ml}$. The FXR LBD was complexed with 2-fold molar of an SRC2-3 peptide (QEPVSPKKKENALLRYLLDKDDTKD) before filter concentration.

Coregulator Binding Assays. The binding of the various coregulator peptide motifs to FXR LBD in response to ligands was determined by AlphaScreen (Amplified Luminescent Proximity Homogeneous) assays using a hexahistidine detection kit from PerkinElmer (Waltham, MA) as described before ( $\mathrm{Li}$ et al., 2005). The hedragonic acid was purchased from BioBioPha (Yunnan, China), whereas the remaining triterpenes were purchased from TargetMol (Shanghai, China). The experiments were conducted with approximately $20-40 \mathrm{nM}$ receptor LBD and $20 \mathrm{nM}$ biotinylated cofactor peptides in the presence of $5 \mu \mathrm{g} / \mathrm{ml}$ donor and acceptor beads in a buffer containing $50 \mathrm{mM}$ 4-morpholinepropanesulfonic acid, $50 \mathrm{mM} \mathrm{NaF}, 0.05 \mathrm{mM}$ 3-[(3-cholamidopropyl)dimethylammonio]-1propanesulfonate, and $0.1 \mathrm{mg} / \mathrm{ml}$ bovine serum albumin, all adjusted to a $\mathrm{pH}$ of 7.4. The peptides with an $\mathrm{N}$-terminal biotinylation are listed as follows: NCoR-1, QVPRTHRLITLADHICQIITQDFAR; NCoR-2, GHSFADPASNLGLEDIIRKALMGSF; SRC1-2, SPSSHSSLTERHKILHRLLQEGSP; SRC2-3, QEPVSPKKKENALLRYLLDKDDT KD; SRC3-3, PDAASKHKQLSELLRGGSG.

Circular Dichroism Spectroscopy. Circular dichroism (CD) measurements were carried out using a Jasco J-8106 spectropolarimeter (JASCO, Tokyo) as previously described (Zhan et al., 2008). The CD spectra were obtained in $10 \mathrm{mM}$ phosphate buffer ( $\mathrm{pH} 7.4$ ) using a cell with a $0.5-\mathrm{cm}$ path length. FXR LBD protein was dialyzed against phosphate buffer (10 mM phosphate, pH 7.4). Gradient concentrations of ligands were added to the protein. After incubation for 2 minutes at room temperature, the CD spectra were measured.

Dual-Luciferase Reporter Assay. HEK-293T cells were maintained in Dulbecco's modified Eagle's medium containing $10 \%$ fetal bovine serum and were transiently transfected using Lipofectamine 2000 (Invitrogen, Carlsbad, CA). All mutant FXR plasmids were created using the Quick-Change site-directed mutagenesis kit (Stratagene, San Diego, CA). Before 24 hours of transfection, 24-well plates were plated $\left(5 \times 10^{4}\right.$ cells per well). For nuclear receptor luciferase reporter assay, the cells were cotransfected with plasmids encoding full-length nuclear receptors and their cognate luciferase reporters as follows: human FXR $\alpha$ with EcRE-Luc, human peroxisome proliferator-activated receptors $(\alpha, \delta$, and $\gamma$ ) with PPRE-Luc, human retinoic acid-related orphan receptors $(\alpha, \beta$, and $\gamma)$ with the Pcp2/RORE-Luc, human glucocorticoid receptor or androgen receptor with the MMTV-Luc, human retinoic acid receptors with $\beta$ RE-Luc reporter. For GPBAR1 reporter assay, the cells were cotransfected with plasmids encoding full-length GPBAR1 and pCRE-luc reporter (Yu et al., 2015). Ligands were added 5 hours after transfection. Cells were harvested 24 hours later for the luciferase assays with the DualLuciferase Reporter assay system (Promega, Madison, WI). The luciferase activities were normalized to renilla activity cotransfected as an internal control.

Crystallization and Structure Determination. The crystal of FXR/hedragonic acid complex was grown at room temperature in hanging drops containing $1.0 \mu \mathrm{l}$ of the ligand-protein solutions and $1.0 \mu \mathrm{l}$ of well buffer containing 0.1 M HEPES (pH 7.5), 10\% (w/v) polyethylene glycol 6000, and 5\% (v/v) (土)-2-methyl-2,4-pentanediol. The crystals were directly flash frozen in liquid nitrogen for data collection. The observed reflections were reduced, merged, and scaled with DENZO and SCALEPACK in the HKL2000 package (HKL Research, Inc., Japan) (Otwinowski and Minor, 1997). The structures were determined by molecular replacement in the CCP4 suite (Collaborative Computational Project, Number 4, San Diego Supercomputer Centre). Manual model building was carried out with Coot (Emsley and Cowtan, 2004), followed by Refmac5 refinement in the CCP4 suite.

Molecular Docking. The structure of GPBAR1 for docking was a gift from Vittorio Limongelli (D'Amore et al., 2014). The docking was carried out using the AutoDock4.2 software package (Scripps Research Institute, La Jolla, CA) (Huey et al., 2007). Grid points of $65 \times$ $80 \times 55$ for GPBAR1 with a $0.375-\AA$ spacing calculated around the binding cavity were deemed as the docking site (D'Amore et al., 2014). Three-dimensional structures of ligands were constructed using the software Discovery Studio (Discovery Studio, version 2.5.5; Accelrys Software Inc., San Diego, CA). Atom types were assigned using the CHARMM force field. Partial charges were added using the Gasteiger algorithm. Energy optimization was carried out to obtain the lowest energy conformation using the steepest descent method under the implicit solvent model with a dielectric constant of 1.0. Visualization was performed using the program Pymol (DeLano Scientific, Palo Alto, CA). Default cutoff values of $4.0 \AA$ for van der Waals and $2.5 \AA$ for 
H-bond interactions were used. Ten conformations were retained for each ligand. Then the docking run consisted of 10 million energy evaluations using the Lamarckian genetic algorithm local search method. Otherwise, default docking parameters were applied.

Animals and Treatments. Male mice at 8 weeks of age were maintained under environmentally controlled conditions with free access to standard chow diet and water. Animal experiments were conducted in the barrier facility of the Laboratory Animal Center, Xiamen University, approved by the Institutional Animal Use and Care Committee of Xiamen University, China. Vehicle $[40 \%(\mathrm{w} / \mathrm{v})$ of 2 -hydroxypropyl- $\beta$-cyclodextrin] or hedragonic acid $(10 \mathrm{mg} / \mathrm{kg}$ body weight) dissolved in vehicle was i.p. injected once daily for 5 days. Six hours after the fifth injection, $500 \mathrm{mg} / \mathrm{kg}$ body weight of acetylpara-aminophenol (APAP) solved in phosphate-buffered saline was i.p. injected into the mice. Twenty four hours later, mice were sacrificed. Parts of liver tissues were fixed in $4 \%$ paraformaldehyde, and the liver histology characterization was analyzed by H\&E staining with paraffinembedded sections by standard procedures. Other liver tissues were collected for real-time quantitative reverse-transcription polymerase chain reaction (PCR). The serums were collected to measure enzyme activities, including lactate dehydrogenase (LDH), alanine aminotransferase (ALT), and aspartate aminotransferase (AST), using commercial kits (Biosino Bio-technology and Science Inc., Beijing, China; Nanjing Jiancheng Bioengineering Institute, Nanjing, China).

PCR. RNA was isolated using an RNA kit (Omega Bio-Tek, Norcross, GA). The first-strand cDNA was obtained by the TAKARA reverse transcription kit (Takara Bio Inc., Kusatsu, Japan). Real-time quantitative PCR was performed on a CFX96 Real-Time PCR Detection System (Bio-Rad, Hercules, CA) using SYBR Premix Ex TaqTM (TAKARA Bio Inc.). Relative mRNA expression levels were normalized to glyceraldehyde-3-phosphate dehydrogenase levels. The primers used in this assay are listed in Supplemental Table 1.

Statistical Analysis. Results are expressed as the mean \pm S.E.M. $P$ values were calculated by using unpaired two-tailed Student's $t$ test. $P$ less than 0.05 was considered significant.

\section{Results}

Identification of Hedragonic Acid as a Novel FXR Agonist. In search of novel modulators for FXR, we used FXR LBD as a bait to screen natural compounds based on the AlphaScreen biochemical assay, which determines the efficacy of small molecules in influencing binding affinity of FXR with coregulator peptides (Jin et al., 2013). The screen results revealed a natural pentacyclic oleanane-type triterpene, hedragonic acid, potently activated FXR in a concentration-dependent manner (Fig. 1A). Hedragonic acid induces FXR to recruit nuclear receptor coactivator motifs from SRC1, SRC2, and SRC3, but not corepressor motifs from NCoR (Fig. 1B), suggesting that hedragonic acid is an agonist of FXR. We further used CD spectroscopy to detect the secondary structural changes of FXR LBD induced by ligands (Fig. 1, C and D). The results showed that hedragonic acid induced conformational changes of FXR LBD in a similar way to the full agonist GW4064 (3-(2,6Dichlorophenyl)-4-(3'-carboxy-2-chlorostilben-4-yl)oxymethyl-5isopropylisoxazole), suggesting the physical interaction of hedragonic acid with FXR. Moreover, cell-based reporter assays further revealed that hedragonic acid selectively activates FXR among many other nuclear receptors tested, but with a relatively moderate activity compared with the synthetic agonist GW4064 (Fig. 1E). Importantly, hedragonic acid induced the expression of FXR target genes in primary hepatocytes from wild-type mice but not FXR knockout mice in a dose-dependent manner, affirming that hedragonic acid is an FXR agonist (Supplemental Figs. 1 and 2).
To investigate whether other oleanane-type triterpenes are FXR ligands, asiatic acid, oleanolic acid, 18 $\beta$-glycyrrhetinic acid, friedelin, ursolic acid, and betulinic acid were selected to test their activities on FXR in recruiting coactivators by AlphaScreen assays. Despite high structural similarity among these triterpenes, none of the tested triterpenes except hedragonic acid activated FXR (Fig. 1F), demonstrating a highly selective structural recognition of oleanane-type triterpenes by FXR. Interestingly, hedragonic acid showed a weak GPBAR1 activity in cell-based reporter assay (Fig. 1G). Structurally compared with other triterpenes, hedragonic acid is characterized by both carbonyl groups on one end in the C3 (Supplemental Fig. 3), which may play a pivotal role in recognition of the pocket-binding sites in FXR.

Structure Determination of the Binding Mode of Hedragonic Acid with FXR. To determine the molecular basis of the binding selectivity of hedragonic acid by FXR, we solved the crystal structure of FXR complexed with hedragonic acid [Fig. 2; Supplemental Table 2, Protein Data Bank identifier (PDB ID) 5wzx]. The data statistics and the refined structures are summarized in Supplemental Table 2. The structure of hedragonic acid-bound FXR LBD assumed a dimer form with the arrangement of a three-layer helical sandwich, with hedragonic acid occupying the pocket formed by the helical structure of H3, H9, and H11 (Fig. 2A), resulting in an active conformation for FXR. Hedragonic acid was clearly observed to fit in the electron density map as shown in Fig. 2B, predominately by hydrophobic interactions with FXR residues of Trp454, Phe329, Met328, Phe366, Leu287, Phe461, and Trp464. In addition, two polar contacts with the C terminus of helix 10 further anchored the ligand position: one is a $2.39-\AA$ hydrogen bond from His 477 to the carbonyl oxygen of hedragonic acid, and the other hydrogen bond is between the NH moiety of Trp454 and a lipid carbonyl group in hedragonic acid with a 2.9-Å distance (Fig. 2B). Notably, these two key pharmacophores are unique features of hedragonic acid that are necessary for its molecular recognition by FXR (Fig. 2C), providing a clue in drug design based on the structure of pentacyclic triterpene. Superposition of structures of hedragonic acid-bound FXR with GW4064-bound FXR (PDB ID 3dct) (Akwabi-Ameyaw et al., 2009) and CDCAbound FXR (PDB ID 4qe6) revealed that hedragonic acid occupies a distinct binding site compared with the classic pocket occupied by GW4064 or CDCA (Fig. 2C). The three ligands also exhibited different orientations in the pockets (Fig. 2, C and D).

Due to the unique binding site of hedragonic acid in FXR, hedragonic acid induces a series of conformational changes in FXR LBD (Fig. 3). Specifically, the loss of the classic hydrogen bond interaction with Arg331 and the unoccupied space around the Phe336 caused helices 5, 3, and 7 to inwardly squeezed, leading to the reduction of FXR pocket size (Fig. 3A). In addition, hedragonic acid binding also induced the conformational changes in internal hydrophobic residues of FXR, including Trp454, Phe461, and Tyr369, in comparison with GW4064- or CDCA-bound FXR LBD (Fig. 3, B-D). Thus, hedragonic acid initiates a hydrophobic interaction between its methyl groups and the benzene ring of FXR Tyr369, in addition to a key hydrogen bond interaction with FXR Trp454, which further echoes the formation of the unique binding pocket of FXR by hedragonic acid. Notably, the retained hydrogen bond between 
A

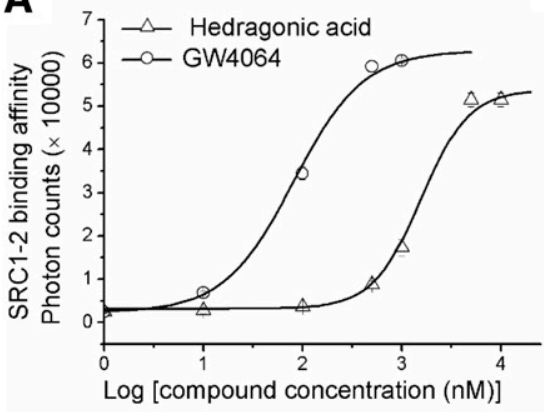

C

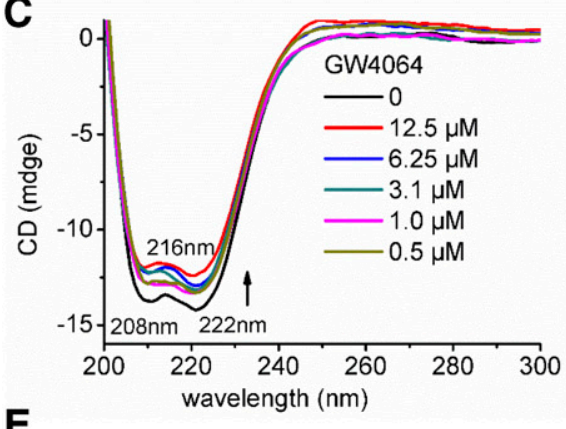

E

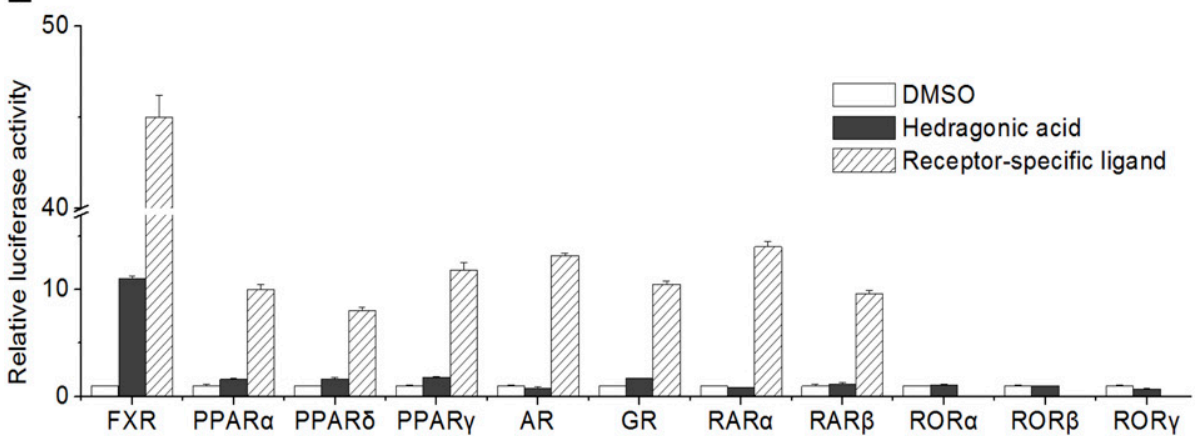

$\mathbf{F}$

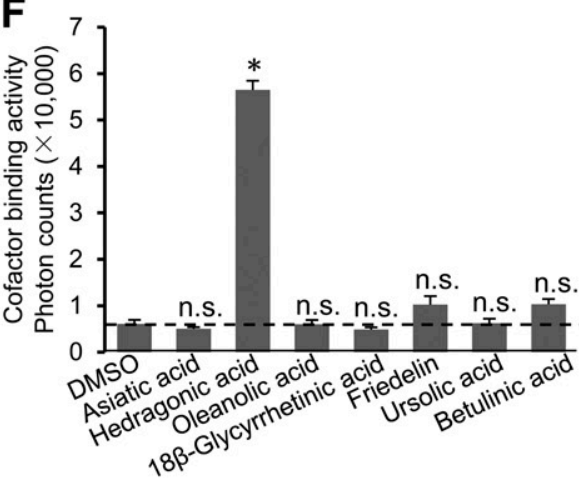

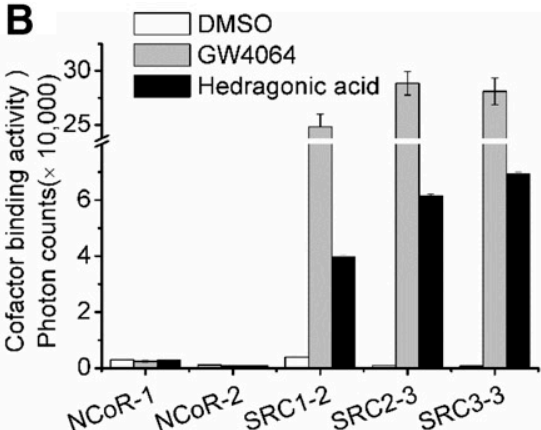

D

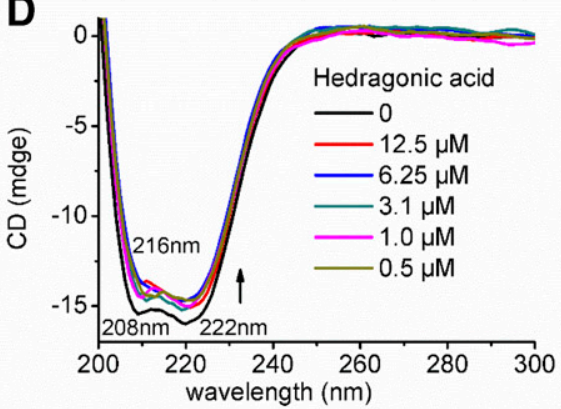

Fig. 1. Identification of natural triterpene hedragonic acid as a novel FXR agonist. (A) Dose responses of compounds in inducing FXR to recruit SRC1-2 coregulator binding motif by AlphaScreen assay. (B) Various coregulator binding motifs bind to FXR in response to $1 \mu \mathrm{M}$ hedragonic acid or GW4064 by AlphaScreen assay. The peptide sequences are listed in the Materials and Methods section. (C and D) Compounds physically bind to the ligand-binding domain of FXR by CD spectroscopy assay. (E) Receptor-specific transactivation by hedragonic acid. HEK-293T cells were cotransfected with plasmids encoding full-length nuclear receptors and their cognate response reporters, respectively (see Materials and Methods section). After transfection, cells were treated with dimethylsulfoxide (DMSO), $1 \mu \mathrm{M}$ hedragonic acid, or ligands specific for each receptor: FXR, $0.5 \mu \mathrm{M}$ GW4064; peroxisome proliferator-activated receptor (PPAR) $\alpha, 1 \mu \mathrm{M}$ GW590735 (2-methyl-2-[4-[[[4-methyl-2[4-(trifluoromethyl)phenyl]-1,3-thiazole5-carbonyl]amino]methyl]phenoxy]propanoic acid); PPAR $\delta, 1 \mu \mathrm{M}$ GW0472 (2-(4-((2-(4(Trifluoromethyl)phenyl)-5-methylthiazol-4-yl)methylthio)-2-methylphenoxy)acetic acid); PPAR $\gamma, 1 \mu \mathrm{M}$ rosiglitazone; androgen receptor (AR), $0.1 \mu \mathrm{M}$ dihydrotestosterone; glucocorticoid receptor (GR), $0.1 \mu \mathrm{M}$ dexamethasone; retinoic acid receptor $\alpha(\operatorname{RAR} \alpha)$ and $\operatorname{RAR} \beta, 1 \mu \mathrm{M}$ all-trans-retinoic acid Data were normalized to renilla activity cotransfected as an internal control. Values are the means \pm S.E.M. of three independent experiments. (F) FXR selectively recognized hedragonic acid from various natural triterpenes to recruit SRC1-2 by AlphaScreen assay; $* P<0.001$ vs. vehicle control; n.s., no significance (Student's $t$ test). (G) The GPBAR1 activities of compounds (5 $\mu \mathrm{M}$ ) by reporter assay; $* P<0.01$ vs. vehicle control (Student's $t$ test). Values are the means \pm S.E.M. of three independent experiments. ROR, retinoic acid-related orphan receptor.

the carbonyl oxygen of hedragonic acid and the $\mathrm{NH}$ moiety in His447 might also play an important role in maintaining the active state of FXR (Fig. 3D), which stabilizes the protein conformation that is capable of recruiting coactivators (Jin et al., 2013).

Structural Validation by Mutagenesis Assays. To validate the roles of the critical residues in the hedragonic acid-binding pocket, site-directed mutagenesis was used to test the transcriptional activity of FXR in response to hedragonic acid in cell-based reporter assays. An A291W mutation was designed to cause a sharp reduction in the size of the FXR pocket through the enlargement of the corresponding side chain in the ligand-binding pocket (Fig. 4, A and B). As expected, a dramatic abolishment in FXR transcriptional activity by GW4064, CDCA, and hedragonic acid was observed in this mutated FXR (Fig. 4D). The H447F mutant was constructed to destroy the hydrogen bonds between His447 and hedragonic acid or CDCA, which are the key interactions in the binding modes of FXR with these two ligands (Fig. 4, A and $\mathrm{C}$ ). Correspondingly, $\mathrm{H} 447 \mathrm{~F}$ resulted in the vanished FXR transcriptional induction by hedragonic acid and CDCA, respectively (Fig. 4D). Considering the unique polar 
A
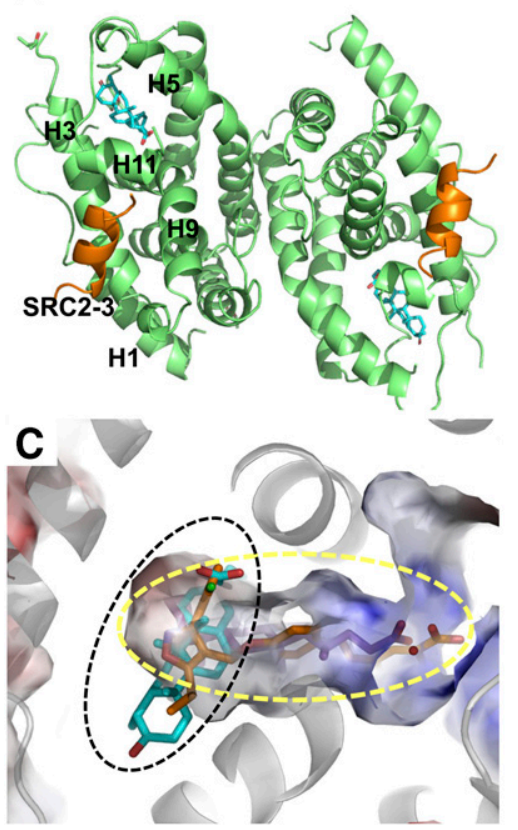

B

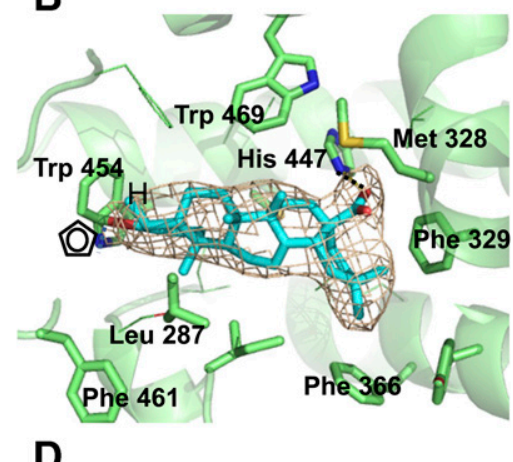

D

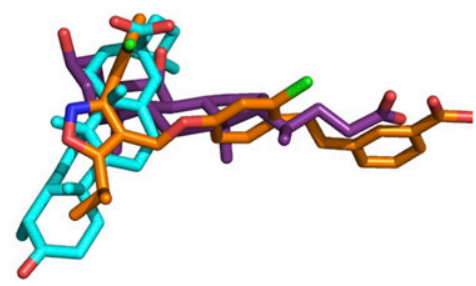

Fig. 2. Structural determination of the FXR LBD in complex with hedragonic acid. (A) The dimer structure of FXR LBD bound with hedragonic acid in ribbon representation. FXR LBD is in light green, and the SRC2-3 motif is in orange. The bound hedragonic acid is shown in stick representation with carbon and oxygen atoms depicted in cyan and red, respectively. (B) $2 \mathrm{Fo}-\mathrm{Fc}$ electron density map $(1.0 \sigma)$ showing the bound hedragonic acid in FXR LBD. (C) Specific location of hedragonic acid in the binding pocket. The unique binding pocket of hedragonic acid is shown in a dashed black circle, and the classic binding pocket is shown in a dashed yellow circle. (D) Superposition of hedragonic acid (cyan) with GW4064 (orange) and CDCA (purple). distribution of the residues around the hedragonic acid-binding pocket, three mutants of L287T, T288V, and F284Y were chosen to evaluate the effect of the polar changes on the specificity of the pocket-binding site. Specifically, the L287T was located in the overlapped binding site of the three ligands in their binding pockets. This mutant changed the favorable hydrophobic environment in the ligand-binding pocket as it occupied a vital position forming hydrophobic interactions with the lipid and bulky steric moiety of ligands (Fig. 4A). Accordingly, the transcriptional activity of FXR with the L287T mutation was abolished by all three ligands (Fig. 4D). In contrast, Thr288 and Phe284 only participated in the binding pocket of hedragonic acid. The lack of a hydroxyl side chain in the T288V mutant weakened electrostatic interactions of FXR with the carbonyl group of hedragonic acid, leading to a moderate decline in the transcriptional activity of FXR by hedragonic acid. In contrast, no changes in transcriptional activity by GW4064 and CDCA were observed in this mutant. Based on our solved structure, a putative hydrogen bond was predicted to emerge between hedragonic acid and the mutant F284Y due to the hydroxyl group of tyrosine as a hydrogen bond donor in the interaction with hedragonic acid. As expected, the FXR transactivation of this mutant was significantly enhanced by hedragonic acid (Fig. 4D), but with a relatively modest impact on the function of CDCA and GW4064. These results further validated the unique binding mode of hedragonic acid with FXR.

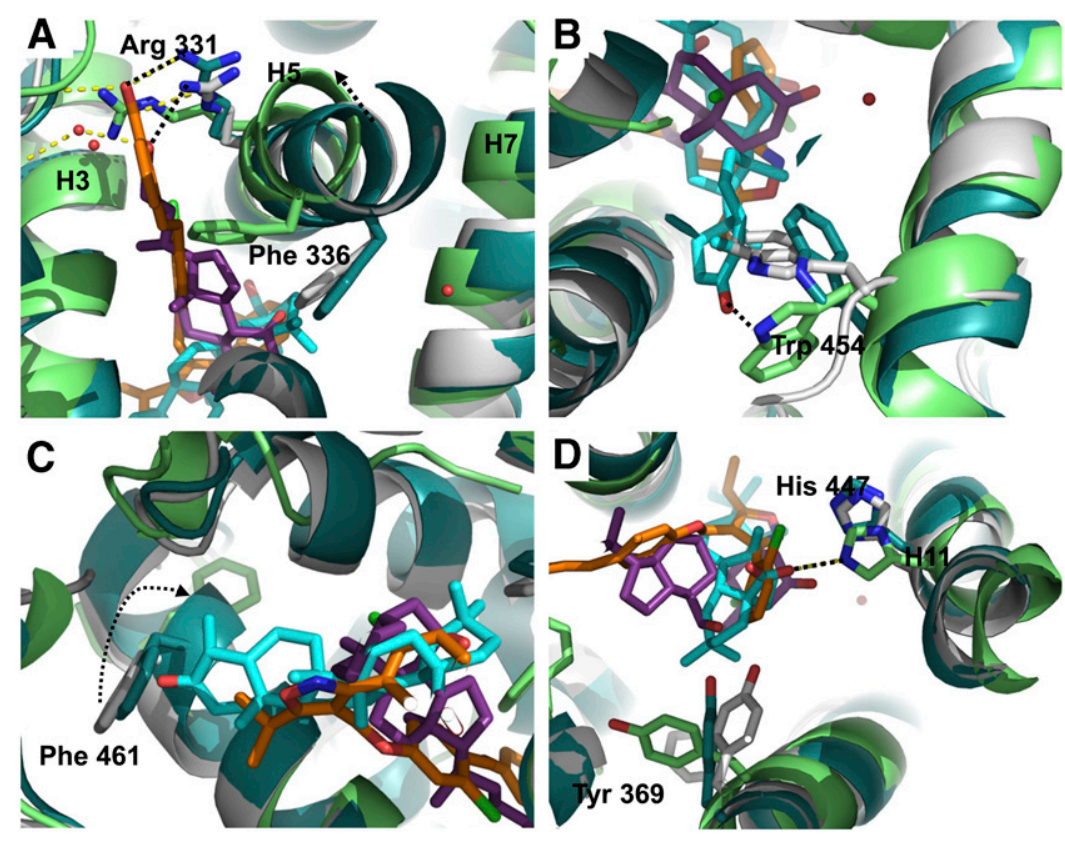

Fig. 3. Conformational changes of FXR LBD induced by ligand binding. Superposition of the FXR/hedragonic acid structure (FXR is in light green and hedragonic acid is in cyan) with the FXR/GW4064 structure (PDB ID 3dct, FXR is in dark green and GW4064 is in orange) and FXR/CDCA (PDB ID 4eq6, FXR is in white and CDCA is in purple). (A) Hedragonic acid binding to FXR reduced the pocket size formed by helices 5,3 , and 7 compared with GW4064 or CDCA. (B-D) Conformational changes of indicated amino acids induced by ligand binding. The conformational changes and hydrogen bonds are shown using dashed arrows and lines, respectively. 


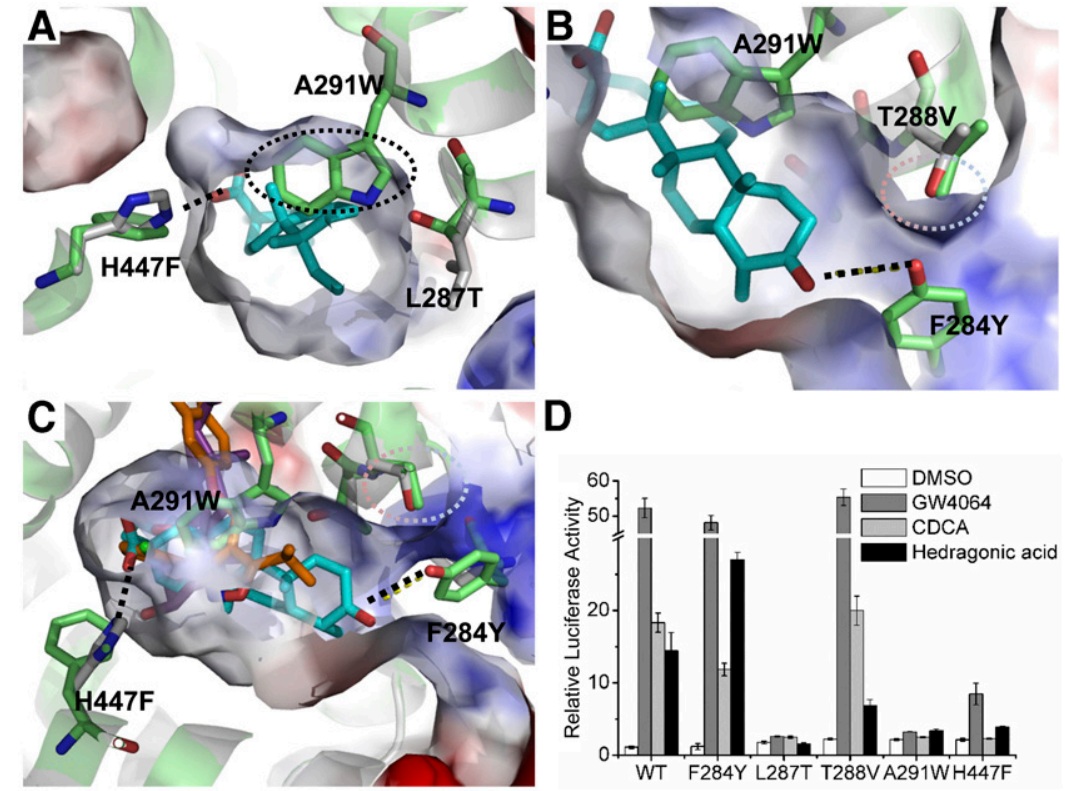

Fig. 4. Functional correlation of the hedragonic acid and FXR interactions. (A-C) Superposition of the FXR/hedragonic acid structure (FXR is in light green) with the FXR/GW4064 structure (PDB ID 3dct, FXR is in dark green and GW4064 is in orange) and FXR/CDCA (PDB ID 4eq6, FXR is in white and CDCA is in purple). The dashed lines indicate the hydrogen bond interaction between His447 and hedragonic acid, and the mutations of A291W and T288V are shown in dashed black circles and dashed gray circles, respectively. (D) Different effects of mutations of key FXR residues on its transcriptional activity in response to hedragonic acid, GW4064, and CDCA in cell-based reporter assays. HEK-293T cells were cotransfected with plasmids encoding full-length wild-type (WT) FXR or FXR mutants as indicated in the figure, together with an EcRE luciferase reporter. The cells were treated with $1 \mu \mathrm{M}$ hedragonic acid, $0.5 \mu \mathrm{M}$ GW4064, and $1 \mu \mathrm{M}$ CDCA. Values are the mean \pm S.E.M. of three independent experiments. DMSO, dimethylsulfoxide.
Structural Comparison of Ligands Binding to FXR and GPBAR1. The obvious difference between hedragonic acid and oleanolic acid is apparent with the ketone for hedragonic acid and the hydroxyl group for oleanolic acid, respectively, at position C-3 (Supplemental Fig. 3), which may dictate the differential binding affinity of oleanane-type triterpenes to FXR and GPBAR1. If the ketone of hedragonic acid is replaced with a hydroxyl group, the volume of van der Waals and polar molecular surface would increase in this orientation of the FXR pocket (Supplemental Fig. 4; Supplemental Table 3). In the ligand-binding pocket of FXR, the cavity formed by key residues of Phe461, Trp454, and Leu287 offers a relatively narrow and hydrophobic space for ligand binding with a 3.1-Å distance between the ketone oxygen atom and the $\mathrm{C} 1$ atom of aromatic face (pi system) in Trp454 (Fig. 5A; Supplemental Fig. 4), which acts as a critical factor in the preference of hedragonic acid from the oleanane-type triterpenes for FXR.

To further investigate the structural mechanism of the binding specificity of oleanane-type triterpenes to FXR and GPBAR1, we compared the two binding modes of the crystal structure of hedragonic acid with FXR and the docking model of oleanic acid with GPBAR1 using AutoDock4.2 (Fig. 5). The docking simulations on the GPBAR 1 in complex with oleanolic acid showed that the oleanolic acid occupies the binding site of GPBAR 1 by more polar interactions than hedragonic acid in FXR (Fig. 5, B and F). In particular, oleanolic acid occupies the cavity formed by TM2, TM3, TM5, and TM7, with its oleananetype triterpenoid scaffold forming favorable interactions with the side chains of Glu169, Tyr240, Phe96, Leu68, and Ser270 (Fig. 5B). Moreover, the hydroxyl group in the C-3 moiety of oleanolic acid extends to the inside position of the hydrophobic cavity formed by Leu24, Leu68, and Trp237, establishing a key hydrogen bond interaction with Ser270 (Fig. 5, B and D). This key hydrogen bond might be unavailable if the C-3 position changes to the ketone of hedragonic acid, resulting in a weaker activity compared to GPBAR1. Also, compared with oleanolic acid, hedragonic acid is characterized by the methyl group at C-4. The steric properties of exocyclic methyl and triterpenoid are important determinants to bind the different shapes of lipophilic pockets. In complex with FXR, the single methyl group at C-4 of hedragonic acid favors its interaction through a cation- $\pi$ interaction with Trp454 with fewer steric effects (Fig. 5, C and E). However, more hydrophobic interactions are acquired for the two methyl groups at C-4 of oleanolic acid when it binds to GPBAR1 (Fig. 5B). Thus, the hydroxyl group and ketone at position C-3 and the steric hindrance of the adjacent groups in the scaffold of triterpenoid determine the preference or selectivity of hedragonic acid and oleanolic acid to FXR and GPBAR1, respectively.

To further elucidate the differential binding modes between oleanolic acid and hedragonic acid to GPBAR1, we mutated several key residues that contribute to the ligand interaction, and then tested the activity of these mutated GPBAR1 in response to oleanolic acid and hedragonic acid in cell-based reporter assays. The mutations $\mathrm{Y} 89 \mathrm{~F}$ and E169A were predicted to damage the polar interaction of ligands (Fig. 5B; Supplemental Fig. 5), and the A67W and L71T mutations were designed to destroy the hydrophobic environment for ligand binding (Fig. 5D; Supplemental Fig. 5). As expected, these mutations substantially reduced GPBAR1 activities mediated by both oleanolic acid and hedragonic acid (Supplemental Fig. 5). The S270A mutation was designed to abolish the hydrogen bond between oleanolic acid and GPBAR1. As expected, S270A substantially decreased the activity of GPBAR1 regulated by oleanolic acid, but not by hedragonic acid (Supplemental Fig. 5). These results further affirm the ligand-binding pocket of GPBAR1 simulated by molecular docking, and highlight the differential roles of GPBAR1 transmembrane residues in recognizing various ligands.

Hedragonic Acid Showed Therapeutic Effects on Liver Injury and Inflammation Dependent on FXR. FXR mediated by ligands plays important roles in liver protection. To investigate the effects of hedragonic acid in liver injury, APAP-induced liver injury was used as a mouse model. After being administered hedragonic acid $(10 \mathrm{mg} / \mathrm{kg})$ for 5 days, mice were i.p. injected with a single challenge of 

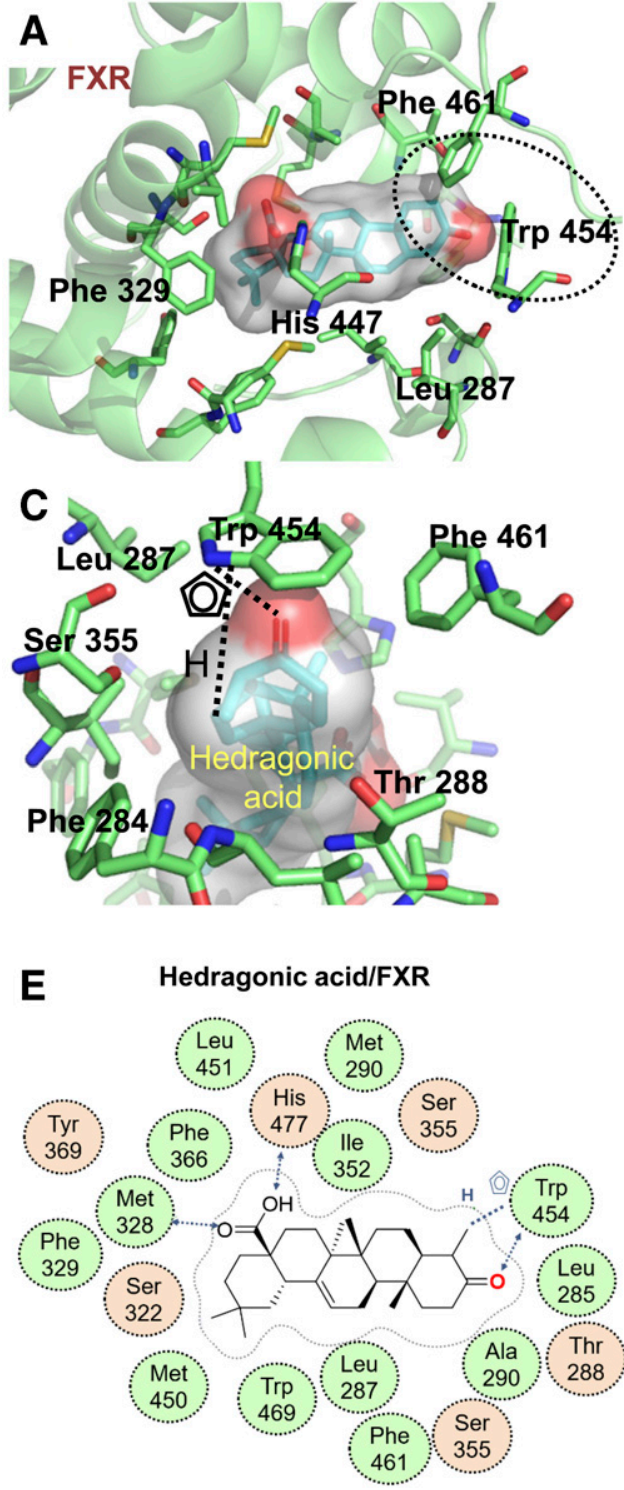
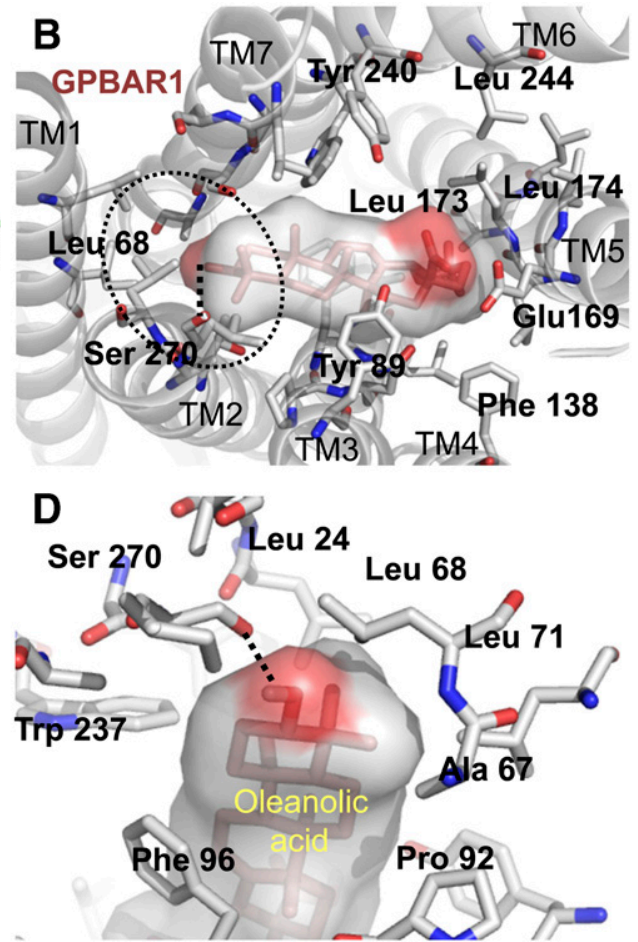

F Oleanolic acid/GPBAR1

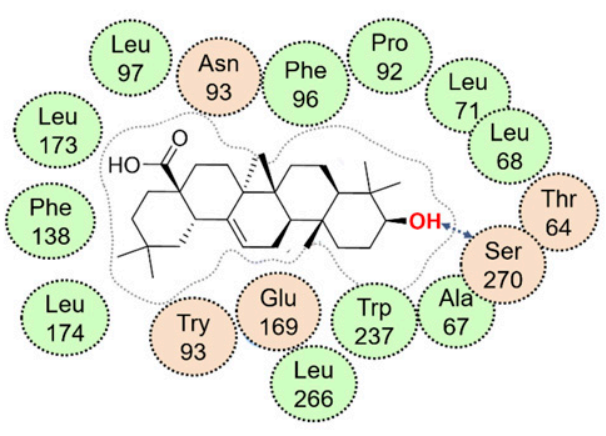

Fig. 5. Structural comparison of ligands binding to FXR and GPBAR1. (A) The crystal structure of hedragonic acid bound with FXR LBD in ribbon representation. FXR LBD is in light green, and hedragonic acid is in light blue. (B) The docking model of oleanolic acid binding to GPBAR1. GPBAR1 is in gray, and oleanolic acid is in pink. The selective positions are marked by a dashed circle. (C and D) Detailed information of binding pocket of FXR bound with hedragonic acid (C) and GPBAR1 bound with oleanolic acid (D). The dashed lines indicate the hydrogen bond interaction between Ser270 and oleanolic acid. (E and F) Schematic representation of FXR-hedragonic acid (E) and GPBAR1-oleanolic acid (F) interactions. The polar residues around the binding pocket are indicated in orange, and the nonpolar ones are in light green. Hydrogen bond interactions are indicated by arrows, and the cation- $\pi$ interaction is marked by symbols.
$\operatorname{APAP}(500 \mathrm{mg} / \mathrm{kg}$ ). Twenty four hours later, liver sections and activities of liver enzymes were examined. H\&E-stained liver sections showed that hedragonic acid pretreatment maintained the liver morphologic characteristics compared with the hepatocyte injury shown in vehicle-treated controls (Fig. 6, A and B). Consistently, serum levels of LDH, ALT, and AST were significantly lower in the hedragonic acid-treated mice compared with those in the vehicle-treated mice (Fig. 6C). It has been reported that ligand-activated FXR could induce several genes involved in xenobiotic metabolism (Lee et al., 2010). We then monitored the mRNA levels of glutathione $S$ transferase $\alpha 3$ and $\alpha 4$, glutathione metabolism-related genes (Gclm and Gpx1), and glucuronosyltransferase (Ugt1a1) in the liver samples of the mice. The results showed that hedragonic acid treatment significantly induced the expression of these genes (Fig. 6D). APAP-induced liver injury also displays liver inflammation due to the increased activities of AST, ALT, and LDH. Hedragonic acid treatment also decreased the mRNA levels of inflammatory genes, including cytokine-inducible nitric oxide synthase, transforming growth factor $\beta$, tumor necrosis factor $\alpha$, cyclooxygenase-2, interleukin $1 \beta$, and MIP-
$1 \alpha$ in liver samples of the mice challenged with overdose APAP (Fig. 6E). These results demonstrate that administration of hedragonic acid could provide protection from APAP-induced liver injury and inflammation.

To investigate whether the liver protection effects of hedragonic acid are dependent on FXR, primary hepatocytes were isolated from wild-type and FXR knockout mice, respectively. After pretreatment with hedragonic acid for 18 hours, $20 \mu \mathrm{g} / \mathrm{ml}$ lipopolysaccharide was administered to the cells. The mRNA levels of the inflammatory genes were measured 6 hours later. The results showed that hedragonic acid significantly decreased the mRNA level of tumor necrosis factor $\alpha$ and interleukin $1 \beta$ in LPS-treated primary hepatocytes from wild-type mice but not in the hepatocytes from FXR knockout mice (Fig. 6F), indicating that hedragonic acid elicits its anti-inflammatory responses by targeting FXR.

\section{Discussion}

Dual or selective FXR and/or GPBAR1 agonists are prevailing strategies in drug discovery for enterohepatic and 

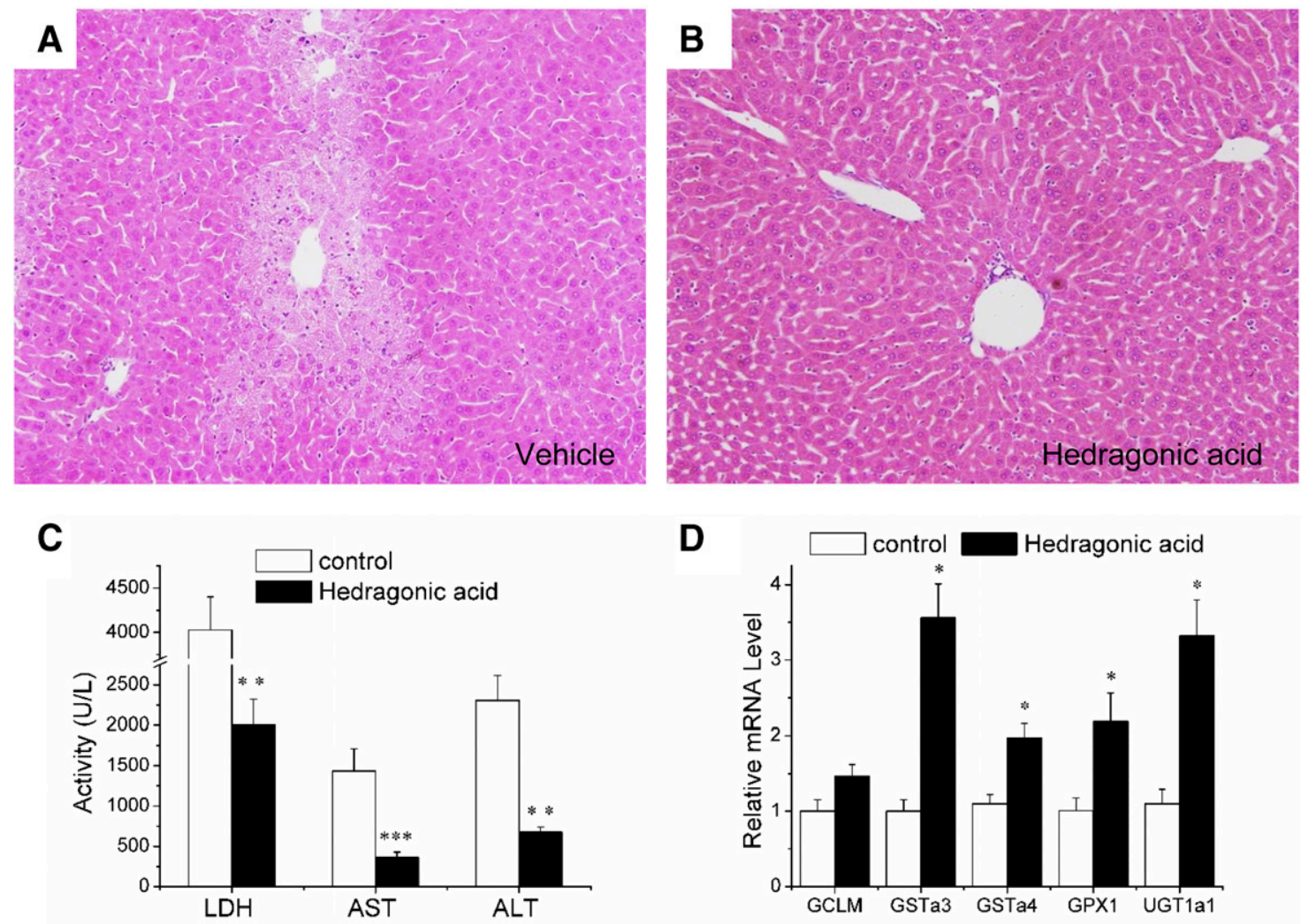

E

F
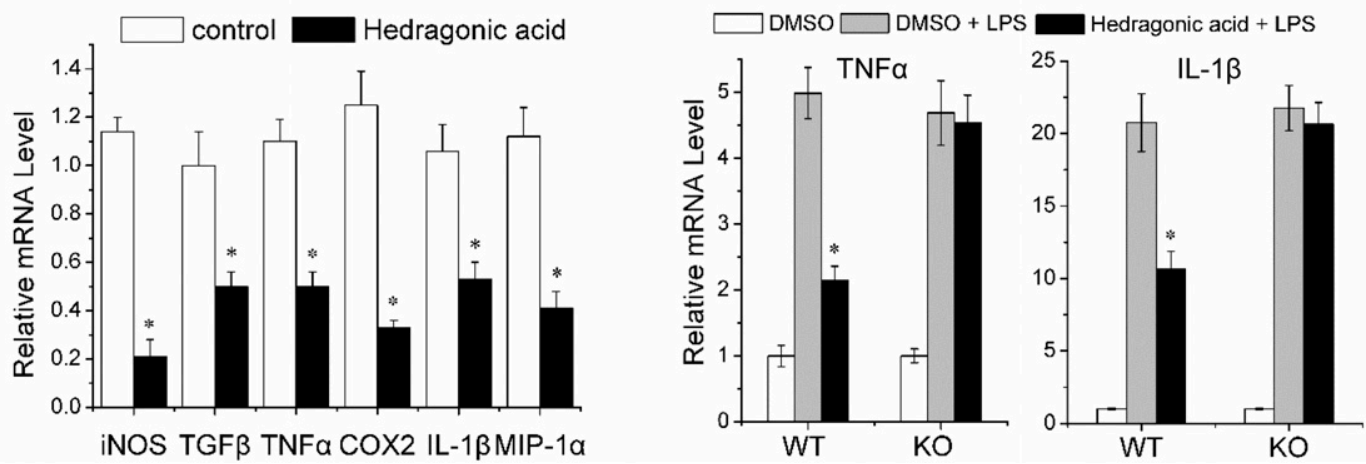

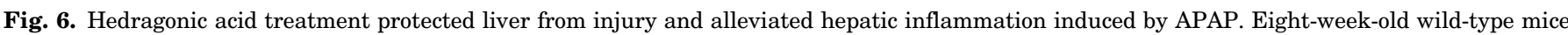

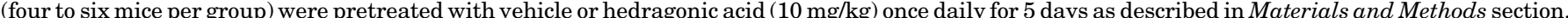

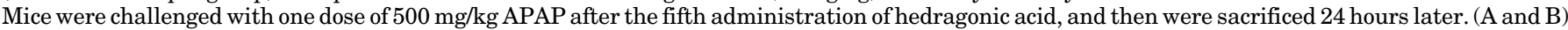

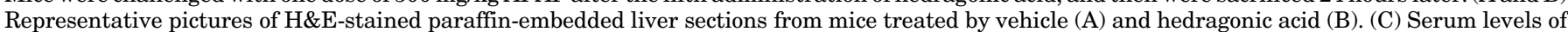

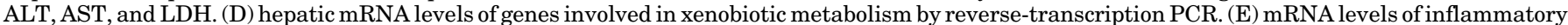

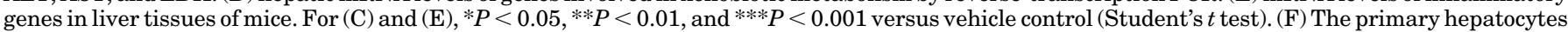

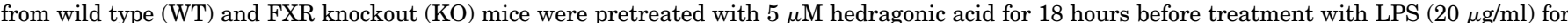

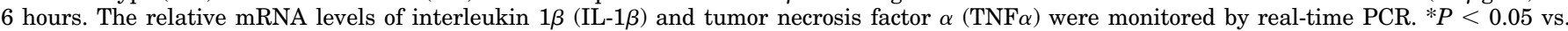

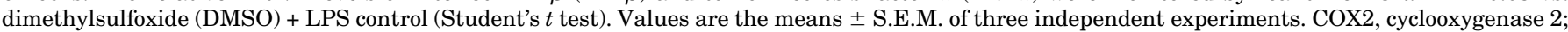
iNOS, cytokine-inducible nitric oxide synthase; MIP-1 $\alpha$, macrophage inflammatory protein $1 \alpha$; TGF $\beta$, transforming growth factor $\beta$.

metabolic disorders (Fiorucci et al., 2009). As endogenous ligands, BAs provide promising templates for structure-based drug design and development. So far, however, only OCA, which is derived from CDCA, has been approved for clinical use for the treatment of primary biliary cholangitis. Notwithstanding that the phase II FLINT trial results demonstrated that OCA is a potential therapy for the nonalcoholic steatohepatitis patient population with diabetes, the severe side effects, including jaundice, worsening ascites, primary biliary cholangitis, reduction in high-density lipoprotein cholesterol, increment in low-density lipoprotein cholesterol, and especially severe pruritus, still exist to limit its clinical usage. Therefore, exploring non-BA agonists for FXR or GPBAR1 might point to a potential direction in drug development by targeting BA receptors. Since series of natural triterpenes have been identified as GPBAR1 agonists, the triterpenes have become promising lead compounds for drug design by targeting GPBAR1. However, it remains unclear why these triterpenes selectively recognize GPBAR1, but not FXR, even though FXR and GPBAR1 share similar binding pockets for 
BAs. In this study, we identified the natural triterpene hedragonic acid as a first oleanane-type triterpene FXR agonist. The crystal structure of hedragonic acid in complex with FXR reveals a unique binding mode with hedragonic acid occupying an unclassical binding site. Structural comparison shed light on the molecular selectivity of similar oleanane-type triterpenes to their preferred receptors, FXR or GPBAR1. In vivo and in vitro studies demonstrate that hedragonic acid exerts its physiologic functions dependent on FXR. These results provide novel evidence for drug design based on natural triterpenes by targeting FXR and/or GPBAR1.

With a great variety of structural compounds, natural products from herbal medicine are an extremely productive source for new medicines or lead compounds for drug discovery and development (Koehn and Carter, 2005). COT is a woody vine of the Celastraceae family, which is widely distributed in Eastern Asia and also North America (Hou, 1955). As a traditional Chinese medicinal herb, the extracts from the stem and root of COT have been used as a remedy against swelling, pain, rheumatoid arthritis, bruises, amenorrhea, vomiting, limbs numbness, hepatitis, Jaundice hepatitis, and cancer (Group CmDW, 1999; Wang et al., 2012). It has been reported that compounds isolated from COT show specific pharmacological functions (Yang et al., 2006; Li et al., 2016; Zhang et al., 2016). However, since numerous and complex compositions exist in plant extracts, the mechanism of multifunctional COT remains unclear. Hedragonic acid is one of the main components of COT (Li et al., 2012) and is identified here as an agonist of nuclear receptor FXR with effective liver protection and anti-inflammatory activity. Considering the important roles of FXR in physiology and pathology, these results suggest that hedragonic acid might be one of the major components of COT for its pharmaceutical uses by targeting FXR.

\section{Acknowledgments}

The authors thank the staff at BL17U of the Shanghai Synchrotron Radiation Source for assistance with data collection. The model of GPBAR1 is a kind gift from Vittorio Limongelli. We also thank Tao Lu for offering the usage of soft wares for molecular docking.

\section{Authorship Contributions}

Participated in research design: L. Jin, Li.

Conducted experiments: Zheng, Lin, Guo, Zhu, Wei, Liu, S. Jin, L. Jin, Li.

Performed data analysis: Lu, L. Jin, Li.

Wrote or contributed to the writing of the manuscript: Lu, L. Jin, Li.

\section{References}

Akwabi-Ameyaw A, Bass JY, Caldwell RD, Caravella JA, Chen L, Creech KL, Deaton DN, Madauss KP, Marr HB, McFadyen RB, et al. (2009) FXR agonist activity of conformationally constrained analogs of GW 4064. Bioorg Med Chem Lett 19:4733-4739.

Benet M, Guzmán C, Pisonero-Vaquero S, García-Mediavilla MV, Sánchez-Campos S, Martínez-Chantar ML, Donato MT, Castell JV, and Jover R (2015) Repression of the nuclear receptor small heterodimer partner by steatotic drugs and in advanced nonalcoholic fatty liver disease. Mol Pharmacol 87:582-594.

Bhushan B, Borude P, Edwards G, Walesky C, Cleveland J, Li F, Ma X, and Apte U (2013) Role of bile acids in liver injury and regeneration following acetaminophen overdose. Am J Pathol 183:1518-1526.

Broeders EP, Nascimento EB, Havekes B, Brans B, Roumans KH, Tailleux A Schaart G, Kouach M, Charton J, Deprez B, et al. (2015) The bile acid chenodeoxycholic acid increases human brown adipose tissue activity. Cell Metab 22: $418-426$.

Castellano JM, Guinda A, Delgado T, Rada M, and Cayuela JA (2013) Biochemical basis of the antidiabetic activity of oleanolic acid and related pentacyclic triterpenes. Diabetes 62:1791-1799.
D’Amore C, Di Leva FS, Sepe V, Renga B, Del Gaudio C, D'Auria MV, Zampella A, Fiorucci S, and Limongelli V (2014) Design, synthesis, and biological evaluation of potent dual agonists of nuclear and membrane bile acid receptors. J Med Chem 57: 937-954.

Emsley P and Cowtan K (2004) Coot: model-building tools for molecular graphics. Acta crystallogr D Biol crystallogr 60 (Pt 12 Pt 1): 2126-2132.

Fiorucci S, Mencarelli A, Palladino G, and Cipriani S (2009) Bile-acid-activated receptors: targeting TGR5 and farnesoid-X-receptor in lipid and glucose disorders. Trends Pharmacol Sci 30:570-580.

Genet C, Strehle A, Schmidt C, Boudjelal G, Lobstein A, Schoonjans K, Souchet M, Auwerx J, Saladin R, and Wagner A (2010) Structure-activity relationship study of betulinic acid, a novel and selective TGR5 agonist, and its synthetic derivatives: potential impact in diabetes. J Med Chem 53:178-190.

Group CmDW (1999) Chinese medicine dictionary, vol 2, pp 1345-1346, Chinese Medical Science and Technology Press, Beijing.

Hou D (1955) A revision of the genus Celastrus. Ann Mo Bot Gard 42:8.

Huey R, Morris GM, Olson AJ, and Goodsell DS (2007) A semiempirical free energy force field with charge-based desolvation. J Comput Chem 28:1145-1152.

Jin L, Feng X, Rong H, Pan Z, Inaba Y, Qiu L, Zheng W, Lin S, Wang R, Wang Z, et al. (2013) The antiparasitic drug ivermectin is a novel FXR ligand that regulates metabolism. Nat Commun 4:1937.

Jin L and Li Y (2010) Structural and functional insights into nuclear receptor signaling. Adv Drug Deliv Rev 62:1218-1226.

Keitel V and Häussinger D (2012) Perspective: TGR5 (Gpbar-1) in liver physiology and disease. Clin Res Hepatol Gastroenterol 36:412-419.

Koehn FE and Carter GT (2005) The evolving role of natural products in drug discovery. Nat Rev Drug Discov 4:206-220.

Kong B, Wang L, Chiang JY, Zhang Y, Klaassen CD, and Guo GL (2012) Mechanism of tissue-specific farnesoid $\mathrm{X}$ receptor in suppressing the expression of genes in bile-acid synthesis in mice. Hepatology 56:1034-1043

Lee FY, de Aguiar Vallim TQ, Chong HK, Zhang Y, Liu Y, Jones SA, Osborne TF, and Edwards PA (2010) Activation of the farnesoid X receptor provides protection against acetaminophen-induced hepatic toxicity. Mol Endocrinol 24:1626-1636.

Li H, Li J, Liu L, Zhang Y, Luo Y, Zhang X, Yang P, Zhang M, Yu W, and Qu S (2016) Elucidation of the intestinal absorption mechanism of celastrol using the Caco-2 cell transwell model. Planta Med 82:1202-1207.

Li J, Yang J, Lu F, Qi Y, Liu Y, Sun Y, and Wang Q (2012) Chemical constituents from the stems of Celastrus orbiculatus. Chin J Nat Med 10 (4): 0279-0283.

Li Y, Choi M, Cavey G, Daugherty J, Suino K, Kovach A, Bingham NC, Kliewer SA, and $\mathrm{Xu} \mathrm{HE}$ (2005) Crystallographic identification and functional characterization of phospholipids as ligands for the orphan nuclear receptor steroidogenic factor- 1 . Mol Cell 17:491-502.

Makishima M, Okamoto AY, Repa JJ, Tu H, Learned RM, Luk A, Hull MV, Lustig KD, Mangelsdorf DJ, and Shan B (1999) Identification of a nuclear receptor for bile acids. Science 284:1362-1365.

Maruyama T, Tanaka K, Suzuki J, Miyoshi H, Harada N, Nakamura T, Miyamoto Y, Kanatani A, and Tamai Y (2006) Targeted disruption of G protein-coupled bile acid receptor 1 (Gpbar1/M-Bar) in mice. J Endocrinol 191:197-205.

Matsubara T, Li F, and Gonzalez FJ (2013) FXR signaling in the enterohepatic system. Mol Cell Endocrinol 368:17-29.

McKenna NJ (2016) Research resources for nuclear receptor signaling pathways. Mol Pharmacol 90:153-159.

Meng Z, Wang Y, Wang L, Jin W, Liu N, Pan H, Liu L, Wagman L, Forman BM, and Huang W (2010) FXR regulates liver repair after CCl4-induced toxic injury. Mol Endocrinol 24:886-897.

Otwinowski Z and Minor W (1997) Processing of X-ray diffraction data collected in oscillation mode. Methods Enzymol 276:307-326.

Parks DJ, Blanchard SG, Bledsoe RK, Chandra G, Consler TG, Kliewer SA, Stimmel JB, Willson TM, Zavacki AM, Moore DD, et al. (1999) Bile acids: natural ligands for an orphan nuclear receptor. Science 284:1365-1368.

Pellicciari R, Gioiello A, Macchiarulo A, Thomas C, Rosatelli E, Natalini B, Sardella R, Pruzanski M, Roda A, Pastorini E, et al. (2009) Discovery of 6alpha-ethyl-23(S)methylcholic acid (S-EMCA, INT-777) as a potent and selective agonist for the TGR5 receptor, a novel target for diabesity. J Med Chem 52:7958-7961.

Pellicciari R, Passeri D, De Franco F, Mostarda S, Filipponi P, Colliva C, Gadaleta RM, Franco P, Carotti A, Macchiarulo A, et al. (2016) Discovery of 3alpha,7alpha,11betaTrihydroxy-6alpha-ethyl-5beta-cholan-24-oic acid (TC-100), a novel bile acid as potent and highly selective FXR agonist for enterohepatic disorders. J Med Chem 59: 9201-9214.

Porez G, Prawitt J, Gross B, and Staels B (2012) Bile acid receptors as targets for the treatment of dyslipidemia and cardiovascular disease. J Lipid Res 53:1723-1737.

Sato H, Genet C, Strehle A, Thomas C, Lobstein A, Wagner A, Mioskowski C, Auwerx J, and Saladin R (2007) Anti-hyperglycemic activity of a TGR5 agonist isolated from Olea europaea. Biochem Biophys Res Commun 362:793-798.

Sun R, Yang N, Kong B, Cao B, Feng D, Yu X, Ge C, Huang J, Shen J, Wang P, et al. (2017) Orally administered berberine modulates hepatic lipid metabolism by altering microbial bile acid metabolism and the intestinal FXR signaling pathway. Mol Pharmacol 91:110-122.

Thomas C, Gioiello A, Noriega L, Strehle A, Oury J, Rizzo G, Macchiarulo A Yamamoto H, Mataki C, Pruzanski M, et al. (2009) TGR5-mediated bile acid sensing controls glucose homeostasis. Cell Metab 10:167-177.

Wagner M, Zollner G, and Trauner M (2011) Nuclear receptors in liver disease. Hepatology 53:1023-1034.

Wang M, Zhang X, Xiong X, Yang Z, Sun Y, Yang Z, Hoffman RM, and Liu Y (2012) Efficacy of the Chinese traditional medicinal herb Celastrus orbiculatus Thunb on human hepatocellular carcinoma in an orthothopic fluorescent nude mouse model. Anticancer Res 32:1213-1220.

Wang YD, Chen WD, Wang M, Yu D, Forman BM, and Huang W (2008) Farnesoid X receptor antagonizes nuclear factor kappaB in hepatic inflammatory response. Hepatology 48:1632-1643. 
Watanabe M, Houten SM, Mataki C, Christoffolete MA, Kim BW, Sato H, Messaddeq N, Harney JW, Ezaki O, Kodama T, et al. (2006) Bile acids induce energy expenditure by promoting intracellular thyroid hormone activation. Nature 439:484-489.

Yang F, Huang X, Yi T, Yen Y, Moore DD, and Huang W (2007) Spontaneous development of liver tumors in the absence of the bile acid receptor farnesoid $\mathrm{X}$ receptor. Cancer Res 67:863-867.

Yang H, Chen D, Cui QC, Yuan X, and Dou QP (2006) Celastrol, a triterpene extracted from the Chinese "Thunder of God Vine," is a potent proteasome inhibitor and suppresses human prostate cancer growth in nude mice. Cancer Res 66 : 4758-4765.

Yu DD, Sousa KM, Mattern DL, Wagner J, Fu X, Vaidehi N, Forman BM, and Huang W (2015) Stereoselective synthesis, biological evaluation, and modeling of novel bile acidderived G-protein coupled bile acid receptor 1 (GP-BAR1, TGR5) agonists. Bioorg Med Chem 23:1613-1628.
Zhan Y, Du X, Chen H, Liu J, Zhao B, Huang D, Li G, Xu Q, Zhang M, Weimer BC, et al. (2008) Cytosporone B is an agonist for nuclear orphan receptor Nur77. Nat Chem Biol 4:548-556.

Zhang Y, Si Y, Zhai L, Guo S, Zhao J, Sang H, Pang X, Zhang X, Chen A, and Qin S (2016) Celastrus orbiculatus Thunb. Reduces lipid accumulation by promoting reverse cholesterol transport in hyperlipidemic mice. Lipids 51:677-692.

Address correspondence to: Dr. Lihua Jin, Room D406, Huang Chao Yang building, Xiamen University, South Xiang An Road, Xiang An District, Xiamen, Fujian 361102, China. E-mail: jinlh@xmu.edu.cn; or Dr. Yong Li, Room D408, Huang Chao Yang building, Xiamen University, South Xiang An Road, Xiang An District, Xiamen, Fujian 361102, China. E-mail: yongli@xmu. edu.cn 